This is the editor's version of the following published document:

Barbero, F. \& S. Villaseñor, E. J. (2015). The thermodynamic limit for black holes in loop quantum gravity. In: The Thirteenth Marcel Grossmann Meeting On Recent Developments in Theoretical and Experimental General Relativity, Astrophysics, and Relativistic Field Theories. Proceedings of the MG13 Meeting on General Relativity. Stockholm University, Sweden, 1-7 July 2012, pp. 2263-2265.

DOI: $10.1142 / 9789814623995 \_0414$

The thermodynamic limit for black holes in loop quantum gravity, Fernando Barbero \& Eduardo J. S. Villaseñor, World Scientific Publishing Co Pte Ltd @ 2015 with permission from World Scientific Publishing Co. Pte. Ltd. http://www.worldscientific.com/worldscibooks/10.1142/9194 


\title{
THE THERMODYNAMIC LIMIT FOR BLACK HOLES IN LOOP QUANTUM GRAVITY
}

\author{
J. Fernando Barbero G. ${ }^{1,3}$ and Eduardo J.S. Villaseñor ${ }^{2,3}$ \\ ${ }^{1}$ Instituto de Estructura de la Materia, CSIC \\ Serrano 123, 28006 Madrid, Spain \\ ${ }^{2}$ Grupo de Modelización, Simulación Numérica y Matemática Industrial \\ Universidad Carlos III de Madrid \\ Avda. de la Universidad 30, 28911 Leganés, Spain \\ ${ }^{3}$ Grupo de Teorías de Campos y Física Estadística \\ Instituto Gregorio Millán, Universidad Carlos III de Madrid \\ Unidad Asociada al Instituto de Estructura de la Materia, CSIC \\ Madrid, Spain
}

March, 2015

\begin{abstract}
This contribution discusses the thermodynamic limit for black holes in loop quantum gravity by using the number-theoretic methods introduced to compute their entropy in this framework. We show how that the subdominant corrections for the entropy in this limit differ from the ones corresponding to the statistical entropy.
\end{abstract}

Key Words: Loop quantum gravity; black holes; thermodynamic limit. 


\section{Black holes and their thermodynamic limit}

The main purpose of this work is to discuss the thermodynamic limit for black holes in loop quantum gravity (LQG), in particular regarding the logarithmic corrections to the Bekenstein-Hawking law. These are important because different models for quantum black holes give different types of corrections (even those that have appeared within LQG itself) and hence they can be used to discriminate between different proposals. An important issue that must be taken into account when studying this problem is the thermodynamic limit [1-3]. The main reason is that the subdominant corrections to the entropy in this limit differ generically from those corresponding to the statistical entropy. The statistical entropy of black holes in LQG differs for the different proposals that have been put forward [4 7 . In particular the solution that can be obtained by using the combinatorial methods developed to deal with the type of problems that crop up in the obtention the statistical entropy 8 - 10 gives

$$
\begin{aligned}
\exp S^{\mathrm{DL}}(a) & =\frac{1}{(2 \pi)^{2} i} \int_{0}^{2 \pi} \int_{x_{0}-i \infty}^{x_{0}+i \infty} \frac{e^{a s}}{s}\left(1-2 \sum_{k=1}^{\infty} e^{-s \sqrt{k(k+2)}} \cos \omega k\right)^{-1} \mathrm{~d} s \mathrm{~d} \omega \\
\exp S^{\mathrm{ENP}}(a) & =\frac{2}{(2 \pi)^{2} i} \int_{0}^{2 \pi} \sin ^{2} \omega \int_{x_{0}-i \infty}^{x_{0}+i \infty} \frac{e^{a s}}{s}\left(1-2 \sum_{k=1}^{\infty} e^{-s \sqrt{k(k+2)}} \frac{\sin ((k+1) \omega)}{\sin \omega}\right)^{-1} \mathrm{~d} s \mathrm{~d} \omega
\end{aligned}
$$

for the Domagała-Lewandowski and the Engle-Noui-Perez proposals respectively [5,7] From these integral representations it is straightforward to obtain the asymptotic behavior of the entropy in the large area limit by studying the analytic structure of the integrands. In particular it is straightforward to derive the Bekenstein-Hawking law. The logarithmic corrections to this behavior originate in the extra integration in $\omega$ that must be performed. This integration must be introduced to deal with the so called projection constraint of the original Ashtekar-Baez-Corichi-Krasnov prescription (in the form introduced by Domagała and Lewandowski) or a similar condition in the Engle-Noui-Perez approach. The logarithmic corrections are respectively given by $-\frac{1}{2} \log a$ in the Domagała-Lewandowski case and $-\frac{3}{2} \log a$ in the Engle-Noui-Perez case.

The preceding expressions for the statistical entropy (coming from a direct state counting) are staircase functions. In order to get suitable smoothed expressions for the entropy it is necessary to modify them. The most appropriate way to do this is by introducing a suitable thermodynamic limit (see, for example, references [1, 2]). It is important to point out that this is not just the large area limit but rather a limit consisting in considering a large system while keeping the relevant intensive parameters constant. The thermodynamic limit can be studied by using both the microcanonical and the canonical ensembles (the result is independent of this choice although the mathematical details involved in the computations differ) and provides and entropy function with the right mathematical properties regarding, in particular, its differentiability and its convexity. Differentiability is very important in order to be able to use the standard formalism of thermodynamics whereas convexity plays a central role in the discussion of thermodynamical stability. If one believes that 
the area ensemble is the right one to describe black holes [11] then their stability hinges precisely in the convexity of the entropy and this, in turn, on the subdominant corrections to the entropy as a function of the area. The best way to get the LQG black hole entropy in the thermodynamic limit is to use the area canonical ensemble [12]. The partition functions can be obtained by relying on the generating functions given in references $[9,13]$. They are

$$
\begin{aligned}
Z^{\mathrm{DL}}(\alpha) & =\frac{1}{2 \pi} \int_{0}^{2 \pi} \frac{\mathrm{d} \omega}{1-2 \sum_{k=1}^{\infty} e^{-s \sqrt{k(k+2)}} \cos \omega k}, \\
Z^{\mathrm{ENP}}(\alpha) & =\frac{1}{\pi} \int_{0}^{2 \pi} \frac{\sin ^{2} \omega \mathrm{d} \omega}{1-2 \sum_{k=1}^{\infty} e^{-s \sqrt{k(k+2)}} \frac{\sin (k+1) \omega}{\sin \omega}} .
\end{aligned}
$$

In the thermodynamic limit the average area and the (smoothed) entropy $\tilde{\sigma}$ are given by

$$
a(\alpha)=-\frac{d}{d \alpha} \log Z(\alpha), \quad \tilde{\sigma}(\alpha):=\alpha a(\alpha)+\log Z(\alpha) .
$$

where the parameter $\alpha$ is conjugate to the area (and, hence, is not an inverse temperature, that would be conjugate to the energy).

In order to obtain the asymptotic behavior of the entropy as a function of the area it is necessary to find the singularities of the integrand in the previous expressions and understand their asymptotic behaviors at them. This can be done as explained in reference [13]. That analysis shows that the Bekenstein-Hawking law holds in the thermodynamic limit, as expected, and also that the subdominant corrections to the large-area behavior of the entropy differ from those obtained for the statistical entropy. In particular, in the Domagała-Lewandowski case the logarithmic correction is $\frac{1}{2} \log a$ whereas in the ENP case the correction is just a constant (see reference 13 for details). The first result is striking because it has a sign opposite to the one obtained for the statistical entropy. Notice, however, that the positive sign that we find in the thermodynamic limit is what one should expect form the theorems of references [1,2]. It is also important to point out that, in the ENP case, the absence of logarithmic corrections does not contradict the general results on the convexity of the entropy but just shows that the corrections for large areas have a different mathematical nature and are very small.

The main conclusion of the work that we have briefly discussed here is the fact that one has to carefully consider the thermodynamical limit in order to understand the subdominant corrections to the entropy behavior. This conclusion should be relevant for every approach to the study of black hole entropy and not only for those inspired in Loop Quantum Gravity.

\section{Acknowledgments}

This work has been supported by the Spanish MICINN research grants FIS200911893, FIS2012-34379 and the Consolider-Ingenio 2010 Program CPAN (CSD2007- 
00042).

\section{References}

[1] J. W. Griffiths, Microcanonical Ensemble in Quantum Statistical Mechanics, J. Math. Phys. 6 (1965) 1447

[2] D. Ruelle, Statistical Mechanics: Rigorous results, (World Scientific, 1999).

[3] R. K. Pathria, Statistical Mechanics, 2nd edition, (Butterworth-Heinemann, 1996)

[4] A. Ashtekar, A. Corichi, and K. Krasnov, Isolated Horizons: the Classical Phase Space, Adv. Theor. Math. Phys. 3 (2000) 419

[5] M. Domagala and J. Lewandowski, Black-hole entropy from quantum geometry, Class. Quant. Grav. 21 (2004) 5233

[6] K. A. Meissner, Black-hole entropy in loop quantum gravity, Class. Quant. Grav. $21(2004) 5245$

[7] J. Engle, K. Noui, A. Perez, and D. Pranzetti, Black hole entropy from the SU(2)-invariant formulation of typeI isolated horizons, Phys. Rev. D 82 (2010) 044050

[8] I. Agullo, J. F. Barbero, J. Diaz-Polo, E. Fernandez-Borja and E. J. S. Villaseñor, Black hole state counting in loop quantum gravity: A number theoretical approach, Phys. Rev. Lett. 100 (2008) 211301

[9] J. F. Barbero G. and E. J. S. Villasenor, Statistical description of the black hole degeneracy spectrum, Phys. Rev. D 83 (2011) 104013

[10] I. Agullo, J. F. Barbero, J. Diaz-Polo, Enrique F. Borja, and E. J. S. Villasenor, Detailed black hole state counting in loop quantum gravity, Phys. Rev. D 82 (2010) 084029

[11] A. Ghosh and A. Perez, Black Hole Entropy and Isolated Horizons Thermodynamics, Phys. Rev. Lett. 107 (2011) 241301

[12] K. V. Krasnov, Geometrical entropy from loop quantum gravity, Phys. Rev. D $\mathbf{5 5 ( 1 9 9 7 ) 3 5 0 5}$

[13] J. F. Barbero G. and E. J. S. Villaseñor, The thermodynamic limit and black hole entropy in the area ensemble, Class. Quantum Grav. 28 (2011) 215014 